\title{
CYANOGEN IN NGC 1851 RED GIANT BRANCH AND ASYMPTOTIC GIANT BRANCH STARS: QUADRIMODAL DISTRIBUTIONS
}

\author{
S. W. Campbell ${ }^{1}$, D. Yong ${ }^{2}$, E. C. Wylie-de Boer ${ }^{2}$, R. J. Stancliffe ${ }^{1,2,3}$, J. C. Lattanzio ${ }^{1}$, G. C. Angelou ${ }^{1}$, \\ V. D’Orazi ${ }^{1,4}$, S. L. Martell ${ }^{5}$, F. Grundahl ${ }^{6}$, and C. SNeden ${ }^{7}$ \\ ${ }^{1}$ Monash Centre for Astrophysics, P.O. Box 28M, Victoria 3800, Australia; simon.campbell@monash.edu \\ ${ }^{2}$ Research School of Astronomy and Astrophysics, Australian National University, Weston, ACT 2611, Australia; david.yong@anu.edu.au \\ ${ }^{3}$ Argelander-Insitut für Astronomie, Universität Bonn, Auf dem Hügel 71, D-53121 Bonn, Germany \\ ${ }^{4}$ Department of Physics \& Astronomy, Macquarie University, Balaclava Rd, North Ryde, Sydney, NSW 2109, Australia \\ 5 Australian Astronomical Observatory, North Ryde, NSW 2113, Australia \\ ${ }^{6}$ Department of Physics and Astronomy, Aarhus University, Ny Munkegade, DK-8000 Aarhus C, Denmark \\ ${ }^{7}$ Department of Astronomy and McDonald Observatory, University of Texas, Austin, TX 78712, USA \\ Received 2012 October 4; accepted 2012 October 29; published 2012 November 15
}

\begin{abstract}
The Galactic globular cluster NGC 1851 has raised much interest since Hubble Space Telescope photometry revealed that it hosts a double subgiant branch. Here we report on our homogeneous study into the cyanogen $(\mathrm{CN})$ band strengths in the red giant branch (RGB) population (17 stars) and asymptotic giant branch (AGB) population (21 stars) using AAOmega/2dF spectra with $R \sim 3000$. We discover that NGC 1851 hosts a quadrimodal distribution of $\mathrm{CN}$ band strengths in its RGB and AGB populations. This result supports the merger formation scenario proposed for this cluster, such that the $\mathrm{CN}$ quadrimodality could be explained by the superposition of two "normal" bimodal populations. A small sample overlap with an abundance catalog allowed us to tentatively explore the relationship between our $\mathrm{CN}$ populations and a range of elemental abundances. We found a striking correlation between $\mathrm{CN}$ and $[\mathrm{O} / \mathrm{Na}]$. We also found that the four $\mathrm{CN}$ peaks may be paired-the two $\mathrm{CN}$-weaker populations being associated with low $\mathrm{Ba}$ and the two $\mathrm{CN}$-stronger populations with high $\mathrm{Ba}$. If true, then s-process abundances would be a good diagnostic for disentangling the two original clusters in the merger scenario. More observations are needed to confirm the quadrimodality and also the relationship between the subpopulations. We also report $\mathrm{CN}$ results for NGC 288 as a comparison. Our relatively large samples of AGB stars show that both clusters have a bias toward $\mathrm{CN}$-weak AGB populations.
\end{abstract}

Key words: globular clusters: general - globular clusters: individual (NGC 1851, NGC 288) - stars: AGB and post-AGB

Online-only material: color figures

\section{INTRODUCTION}

Galactic globular clusters (GCs) are no longer thought to be perfectly homogeneous, simple stellar populations. Although almost all are chemically homogeneous with respect to Fe and heavier elements (omega Cen, M22, Terzan 5, and NGC 1851 being exceptions), it has long been known that GCs show large star-to-star abundance variations for light elements (e.g., C, $\mathrm{N}, \mathrm{O}, \mathrm{Na}, \mathrm{Mg}$, and $\mathrm{Al}$; see reviews by Kraft 1994; Gratton et al. 2004, 2012a). These inhomogeneities are considered anomalous because they are seen in very few halo field stars of similar metallicity (Gratton et al. 2000; Martell \& Grebel 2010). Studies of all phases of evolution, including the red giant branch (RGB), main sequence (MS), and subgiant branch (SGB; e.g., in NGC 6752, Gratton et al. 2001), have shown the same anomalies. This suggests that many of the abundance variations arose in the early phases of cluster evolution.

Recently it was discovered that the globular cluster NGC 1851 has a double SGB, whereby two evolutionary sequences are clearly visible (Hubble Space Telescope photometry; Milone et al. 2008, 2009). The RGB has also been shown to split into two when using particular filters (Han et al. 2009). Spectroscopic observations of this cluster show bimodality in $s$-process abundances (Yong \& Grundahl 2008; Villanova et al. 2010; Gratton et al. 2012c) and a small spread in $[\mathrm{Fe} / \mathrm{H}]$ (rms scatter $\sim 0.05$ dex; Carretta et al. 2011). Gratton et al. (2012c) report that the two SGB populations have slightly different heavy-element contents on average. Carretta et al. (2011) find they can split their RGB sample into a metal-rich and metal-poor population based on the $\mathrm{Fe}-\mathrm{Ba}$ plane, and that each population has its own $\mathrm{O}-\mathrm{Na}$ anticorrelation. This ties in well with the horizontal branch (HB) observations of Gratton et al. (2012b), who also report two separate $\mathrm{O}-\mathrm{Na}$ anticorrelations. As an explanation for these abundance anomalies, as well as the bimodal HB, it has been suggested that NGC 1851 may be a product of a merger between two GCs (van den Bergh 1996; Catelan 1997; Carretta et al. 2010). Bekki \& Yong (2012) recently showed that a merger scenario for NGC 1851 is dynamically plausible. For reviews on the phenomenon of multiple populations in GCs, see, e.g., Piotto (2009), Martell (2011), and Gratton et al. (2012a).

One of the first inhomogeneities discovered in GCs was that of the molecule cyanogen (CN; often used as a proxy for nitrogen). A picture of "CN bimodality" emerged in the 1970s and 1980s (Hesser 1978; Norris \& Freeman 1979; Cottrell \& Da Costa 1981) whereby stars in one population show weak absorption by $\mathrm{CN}$ ("CN-weak" stars) and stars in the other show strong absorption by $\mathrm{CN}$ ("CN-strong" stars). This has been observed in most, if not all, clusters. With the recent interest in NGC 1851 there have been a couple of studies of CN, on the MS (Pancino et al. 2010) and the two SGBs (Lardo et al. 2012). There does, however, appear to be a dearth of studies of $\mathrm{CN}$ in giants in NGC 1851-here we report on observations focusing on CN band strengths in the RGB and asymptotic giant branch (AGB) stars of NGC 1851. 
Table 1

List of Target Stars for NGC 1851

\begin{tabular}{|c|c|c|c|c|c|c|c|}
\hline Type & ID (Walk.) & ID (2MASS) & ID (Carr.) & $V$ & $B-V$ & $S(3839)$ & $\delta S(3839)$ \\
\hline RGB & 24 & $05134832-4003151$ & & 15.40 & 1.00 & 0.31 & 0.19 \\
\hline RGB & 28 & 05134897-4001199 & 41113 & 15.71 & 0.94 & 0.34 & 0.26 \\
\hline RGB & 71 & $05135414-4003038$ & 32112 & 15.45 & 0.98 & 0.73 & 0.62 \\
\hline RGB & 79 & $05135462-4005094$ & $\ldots$ & 15.29 & 1.00 & 0.15 & 0.01 \\
\hline RGB & 120 & $05135671-4001016$ & $\ldots$ & 15.00 & 1.06 & 0.57 & 0.40 \\
\hline RGB & 151 & $05135828-3959586$ & $\ldots$ & 16.19 & 0.90 & 0.43 & 0.41 \\
\hline RGB & 160 & $05135866-4000178$ & $\ldots$ & 16.14 & 0.89 & 0.42 & 0.40 \\
\hline RGB & 161 & $05135867-4000120$ & 44803 & 14.91 & 1.07 & 0.39 & 0.20 \\
\hline RGB & 162 & $05135862-3959242$ & 46228 & 16.19 & 0.91 & 0.11 & 0.09 \\
\hline RGB & 208 & $05135977-4001374$ & $\ldots$ & 15.66 & 0.93 & 0.42 & 0.33 \\
\hline RGB & 368 & $05140259-4000220$ & 44414 & 15.62 & 0.95 & 0.33 & 0.24 \\
\hline RGB & 441 & $05140365-4001596$ & $\ldots$ & 16.18 & 0.89 & 0.28 & 0.26 \\
\hline RGB & 1028 & $05141052-3958095$ & 47385 & 15.41 & 1.00 & 0.12 & 0.00 \\
\hline RGB & 1256 & 05141724-4002080 & 37070 & 14.38 & 1.21 & 0.34 & 0.08 \\
\hline RGB & 1284 & $05141956-4004055$ & 26801 & 14.39 & 1.18 & 0.63 & 0.37 \\
\hline RGB & 1286 & $05141947-4000076$ & 44939 & 14.95 & 1.09 & 0.24 & 0.06 \\
\hline RGB & 1323 & $05142281-4001551$ & 38215 & 16.19 & 0.89 & 0.58 & 0.56 \\
\hline AGB & 182 & 05135918-4002496 & $\ldots$ & 14.93 & 0.92 & 0.35 & 0.17 \\
\hline AGB & 222 & $05140019-4002291$ & $\ldots$ & 14.69 & 0.98 & 0.39 & 0.17 \\
\hline AGB & 245 & $05140068-4003239$ & 30315 & 14.53 & 1.08 & 0.24 & 0.00 \\
\hline AGB & 430 & $05140355-4002499$ & $\ldots$ & 15.03 & 0.95 & 0.16 & -0.01 \\
\hline AGB & 506 & $05140446-4003113$ & $\ldots$ & 14.76 & 0.88 & 0.29 & 0.08 \\
\hline AGB & 572 & $05140508-4002278$ & $\ldots$ & 15.56 & 0.82 & 0.31 & 0.21 \\
\hline AGB & 633 & 05140584-4002126 & $\ldots$ & 14.25 & 1.16 & 0.26 & -0.01 \\
\hline AGB & 680 & $05140659-4002026$ & $\ldots$ & 14.78 & 1.04 & 0.44 & 0.24 \\
\hline AGB & 697 & $05140701-4003449$ & $\ldots$ & 14.73 & 1.02 & 0.17 & -0.04 \\
\hline AGB & 741 & $05140758-4003164$ & $\ldots$ & 15.50 & 0.83 & 0.22 & 0.11 \\
\hline AGB & 848 & $05140883-4002380$ & $\ldots$ & 14.23 & 1.15 & 0.23 & -0.05 \\
\hline AGB & 849 & $05140900-4004539$ & $\ldots$ & 14.83 & 0.97 & 0.41 & 0.21 \\
\hline AGB & 887 & $05140916-4002296$ & $\ldots$ & 14.99 & 0.87 & 0.27 & 0.09 \\
\hline AGB & 988 & $05141034-4004235$ & $\ldots$ & 14.76 & 0.98 & 0.70 & 0.49 \\
\hline AGB & 989 & $05141026-4003150$ & $\ldots$ & 15.42 & 0.80 & 0.17 & 0.05 \\
\hline AGB & 1004 & $05141057-4003308$ & $\ldots$ & 14.93 & 0.94 & 0.33 & 0.15 \\
\hline AGB & 1014 & $05141035-3958148$ & $\ldots$ & 14.72 & 1.04 & 0.57 & 0.36 \\
\hline AGB & 1037 & $05141084-4001475$ & $\ldots$ & 15.19 & 0.88 & 0.52 & 0.37 \\
\hline AGB & 1172 & $05141351-4003408$ & $\ldots$ & 14.87 & 0.96 & 0.66 & 0.47 \\
\hline AGB & 1214 & $05141501-4003040$ & $\ldots$ & 14.85 & 0.97 & 0.39 & 0.20 \\
\hline AGB & 1246 & $05141641-4002214$ & $\ldots$ & 15.42 & 0.80 & 0.29 & 0.17 \\
\hline
\end{tabular}

Notes. IDs (column 2), $V$ magnitudes and $B-V$ values are from Walker (1992). Column 4 IDs are from Carretta et al. (2011) and show the overlap between studies. In column 7 are our raw $\mathrm{CN}$ band strength measurements, $S(3839)$, and in column 8 our de-trended $\mathrm{CN}$ index values $\delta S(3839)$.

\section{STELLAR SAMPLE, OBSERVATIONS, AND DATA REDUCTION}

Our stellar sample was taken from the $B V$ photometry catalog of Walker (1992). This catalog was chosen because the photometry is precise enough to distinguish between the RGB and AGB populations and because it provides accurate astrometry, an important feature for multi-fiber spectroscopy. The chosen sample of stars was cross-matched with the Two Micron All Sky Survey (2MASS) catalog (Skrutskie et al. 2006), thus all of our sample stars are actually 2MASS objects, with positions accurate to $\sim 0$ '.2. Since the two giant branches merge in the color-magnitude diagram (CMD) at higher luminosities, we limited our RGB and AGB samples to $V>14.2$. We show our program stars against the Walker (1992) CMD in Figure 1 and provide a list in Table 1.

Our observational data were taken in 2009 on September 5, 7, 8, and 9 at the Anglo-Australian Telescope using the multifiber spectrograph, AAOmega/2dF (Lewis et al. 2002; Saunders et al. 2004; Sharp et al. 2006). A total of $9 \mathrm{hr}$ of exposures

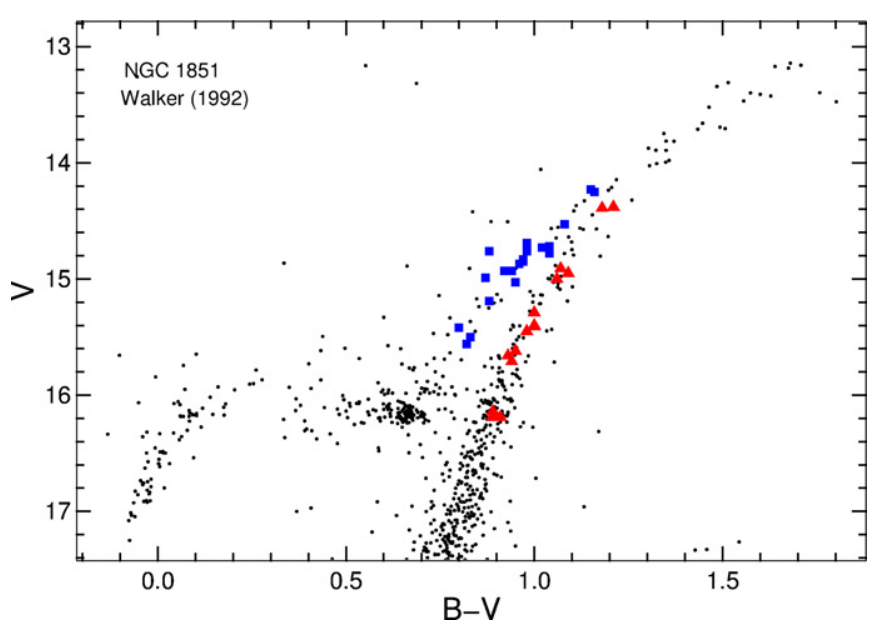

Figure 1. Stellar sample. Small dots are all the stars from the Walker (1992) CMD. Filled triangles (red) are our sample of 17 RGB stars, filled squares (blue) are our 21 AGB stars.

(A color version of this figure is available in the online journal.) 


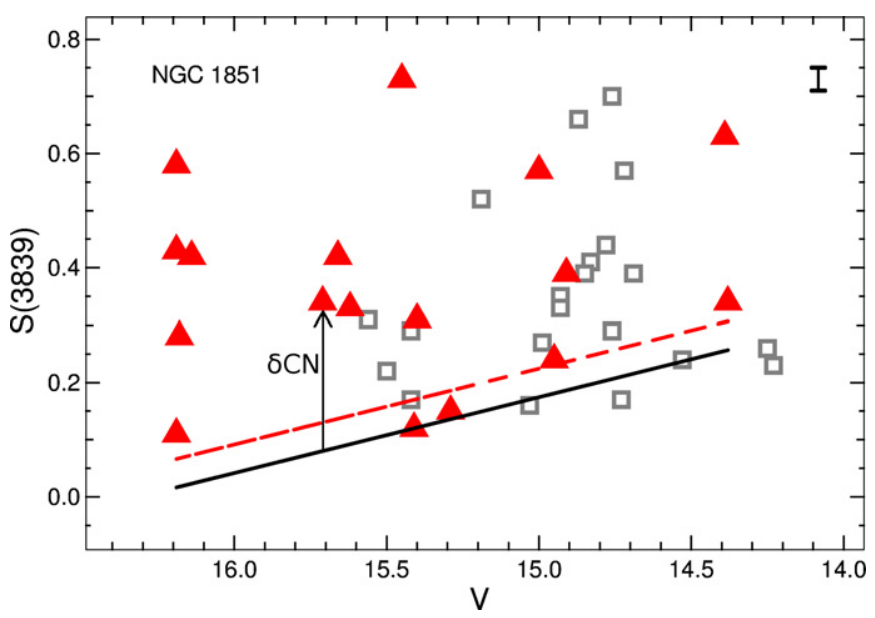

Figure 2. Measured $S(3839) \mathrm{CN}$ index vs. magnitude for the NGC 1851 stars. Filled triangles (red) are RGB stars and open squares (gray) are AGB stars. The dashed line shows a least-squares fit to the five RGB stars at the lower envelope of the distribution. The solid line is the same but offset so that the zero level of $\delta S(3839)(\delta \mathrm{CN})$ is coincident with the star with the lowest $\delta \mathrm{CN}$. The definition of $\delta \mathrm{CN}$ is shown by the arrow. A characteristic error bar for $S(3839)$ is shown at top right (see text for details).

(A color version of this figure is available in the online journal.)

were taken, using three field plate setups. The 1700B grating was used on the blue arm of the spectrograph, which gave a spectral coverage of $3755-4437 \AA$ and includes the violet $\mathrm{CN}$ bands around $3850-3880 \AA$. Spectral resolution in this region was $R \sim 3000$.

Data reduction was carried out using the $2 \mathrm{dF}$ pipeline software $2 d F d r$ (version 3.211, 2009 April) provided by the AAO. Tram-map fits to the multiple spectra from each plate were checked by eye, as were the arc reductions and final reduced science spectra. Our final sample of spectra contained 17 RGB and 21 AGB stars.

To quantify the $\mathrm{CN}$ band strengths in each spectrum, we used the $S(3839) \mathrm{CN}$ index of Norris et al. (1981), which compares a section of the $\mathrm{CN}$ bands with a neighboring pseudo-continuum (Equation 1). IRAF was used to measure the integrated fluxes of Equation (1) for all the program stars:

$$
S(3839)=-2.5 \log \frac{\int_{3846}^{3883} I_{\lambda} d \lambda}{\int_{3883}^{3916} I_{\lambda} d \lambda} .
$$

\section{RESULTS AND DISCUSSION}

In Table 1 we list the $S(3839)$ measurements for each star, while in Figure 2 we plot them versus $V$ magnitude. $\mathrm{CN}$ absorption is known to have a temperature dependence, so we have "de-trended" the data in the same manner as in previous CN studies (e.g., Norris et al. 1981; Ivans et al. 1999; Martell et al. 2008) by fitting a line to the lower envelope of the observations. The value $\delta S(3839)$ (hereafter $\delta \mathrm{CN}$ ) is then the distance from this line to each data point. The resultant $\delta \mathrm{CN}$ values are shown in the lower panel of Figure 3 and listed in Table 1. Errors in wavelength calibration or Doppler offsets due to velocity dispersion were checked and found to be of the order of $\sim 10^{-3}$ in $\delta \mathrm{CN}$. The much larger characteristic error bar for $S(3839)$ given in the figures $( \pm 0.02)$ reflects the typical differences between measurements of $S(3839)$ in two observations of the same star. These pairs of observations were taken on different nights and with different field plates (other clusters in our broader observational campaign were used for this, but the data were taken during the same time frame as for NGC 1851). We found that this was by far the largest source of error. This is probably to be expected since it reflects the combination of many sources of error, including the uncertainties of fiber placement, fiber throughput, slight pointing errors, seeing variability, as well as errors in the data reduction (for example).

In the upper panel of Figure 3, we show a kernel density estimate (KDE) histogram of $\delta \mathrm{CN}$ based on a Gaussian kernel with a bandwidth of 0.035 . This bandwidth was found to be optimal taking into account the error bars and small number statistics. It was tested with many other similar data sets (S. W. Campbell et al., in preparation). It can be seen in Figure 3 that the distribution of $\delta \mathrm{CN}$ in the RGB stars is quadrimodal, having four peaks. Changing the KDE bandwidth within reasonable limits $(\sim 0.02 \rightarrow 0.05)$ does not alter this result. This was an unexpected result because most clusters in our greater sample and in the literature show bimodal distributions. As a comparison, in the right-hand panels of Figure 3 we show the same plot but for NGC 288. The data for this cluster were taken in the same observing run and with the same instrument as our NGC 1851 data. We chose this cluster for comparison because it has a similar metallicity to NGC $1851([\mathrm{Fe} / \mathrm{H}] \sim-1.3$ versus -1.2 , respectively) but a different HB morphology-NGC 1851 has a bimodal HB morphology while NGC 288 has a blue $\mathrm{HB}$ only. As can be seen in this figure, we find the standard bimodality in $\delta \mathrm{CN}$ in the RGB population of NGC 288 .

There is the possibility that the observed quadrimodality in our sample of NGC 1851 RGB stars is a chance occurrence due to a small sample size. A small random sample drawn from a bimodal distribution may give this result. To estimate the likelihood of this happening, we conducted the following test. We established an arbitrary $\delta \mathrm{CN}$ distribution consisting of two Gaussians with centers at 0.05 and 0.45 and FWHMs of 0.10 . Samples of 17 stars were then randomly drawn from this distribution and the pseudo-data was then smoothed with a Gaussian kernel having a bandwidth of 0.035 . We repeated this 1000 times. The results from this test indicate that a small fraction $(\sim 2.5 \%)$ of the randomly generated samples do present quadrimodal distributions (although only 1 out of 1000 was as clearly defined as in the real data). Therefore, we cannot eliminate the small probability that the observed $\delta \mathrm{CN}$ distribution in the RGB population has been drawn from a bimodal population through chance. However, the case for quadrimodality is strengthened by the AGB results in Figure 3-the AGB population also appears to be quadrimodal in $\delta \mathrm{CN}$. Here the stars are distributed differently though, with the majority of the AGB stars being in the two CN-weaker subpopulations, as is usually the case in GCs (e.g., NGC 288 in Figure 3; Norris et al. 1981; Campbell et al. 2011). The probability for attaining a quadrimodal distribution in the RGB population and simultaneously in the AGB population by chance then becomes extremely small, since they are essentially independent populations with different internal distributions of $\delta \mathrm{CN}$.

It has been suggested that NGC 1851 may be a merger product between two GCs, initially as an explanation for producing its bimodal HB (van den Bergh 1996; Catelan 1997). In this scenario it would also be expected that each merging population would have two "normal" subpopulations, each with its own $\mathrm{C}-\mathrm{N}$ and $\mathrm{O}-\mathrm{Na}$ (and possibly $\mathrm{Mg}-\mathrm{Al}$ ) anticorrelations, and that the superimposition of these populations would present dual anticorrelations. In the RGB study by Carretta et al. (2011), it is 


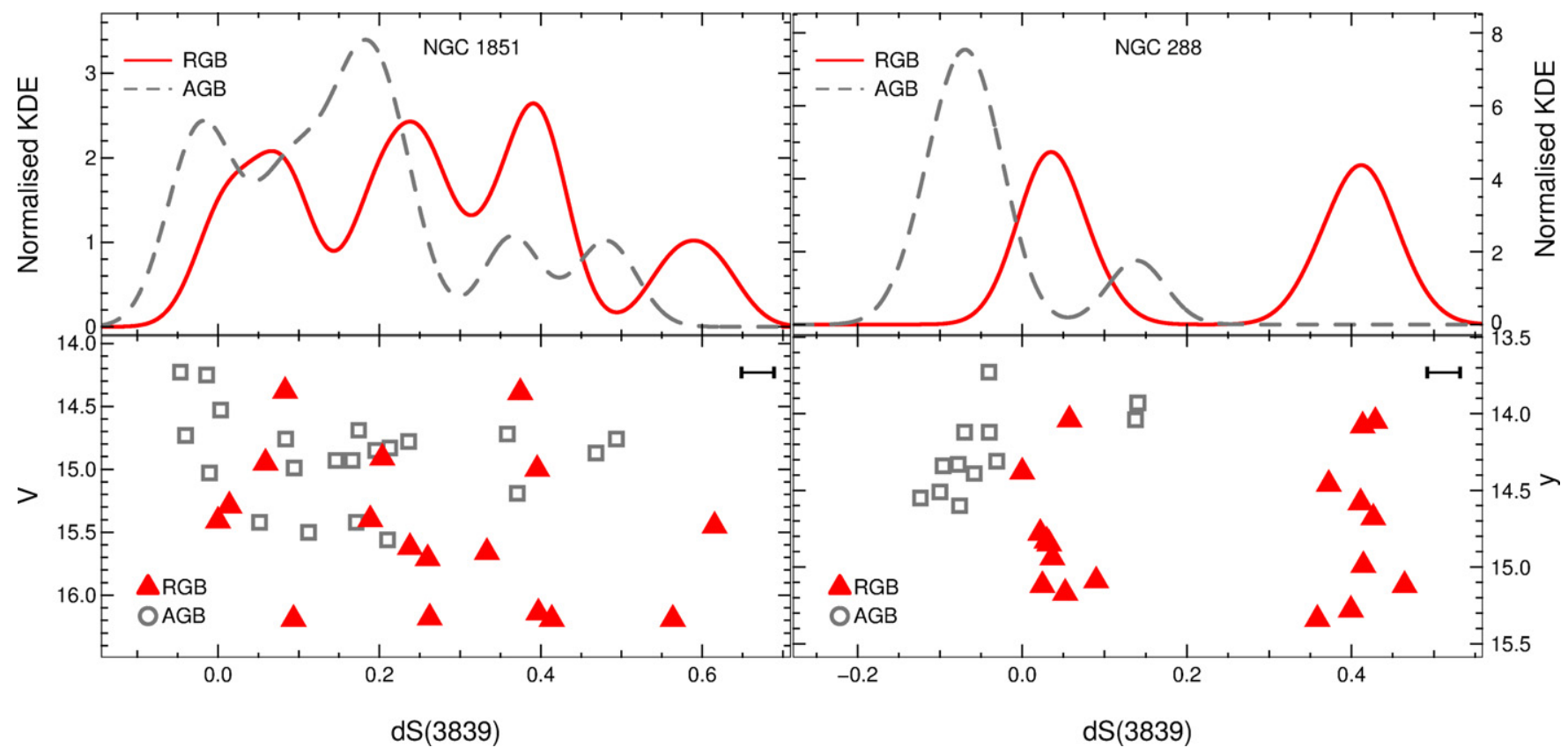

Figure 3. Left-hand bottom panel: $\delta S(3839) \mathrm{CN}$ index vs. $V$ magnitude for NGC 1851 (see Figure 2 for the definition of $\delta S(3839)$ ). Left-hand upper panel: normalized kernel density estimate histogram (Gaussian kernel, bandwidth $=0.035)$ of $\delta S(3839)$. A clear CN quadrimodality can be seen on the RGB and the AGB. A characteristic error bar for $S(3839)$ is shown at top right of the lower panel. Right-hand panels: same as left-hand panels but for NGC 288, for comparison. The bandwidth for the Gaussian kernel is the same as that used for the NGC 1851 data. In this case, photometry (uvby) for the stellar sample selection was provided by F. Grundahl (2009, private communication; Grundahl et al. 1999).

(A color version of this figure is available in the online journal.)

indeed found that NGC 1851 has two $\mathrm{Na}-\mathrm{O}$ anticorrelations, one in their metal-rich population and one in their metal-poor population. This ties in well with the findings of the Gratton et al. (2012b) study where it was found that there are also two independent $\mathrm{Na}-\mathrm{O}$ anticorrelations on the $\mathrm{HB}-$ one in the Red Horizontal Branch (RHB) population and one in the Blue Horizontal Branch (BHB) population. With regards to C and $\mathrm{N}$ the picture is less clear. Lardo et al. (2012) studied the SGB populations and found a spread in $\mathrm{C}$ and $\mathrm{N}$ between stars. These elements were also found to be anticorrelated; however, no bimodal signature was detected. The resolution of the spectra in that study was, however, quite low, with $R \sim 1000$. In the SGB study of Gratton et al. (2012c), it was found that the two SGBs have different average $\mathrm{C}$ abundances. Interestingly, they also found that there are different proportions of $\mathrm{C}$-normal and C-poor stars in each SGB, which may indicate that each SGB hosts multiple subpopulations, again suggestive of a merger scenario. In a study of the two RGB populations, Villanova et al. (2010) found a spread in CNO elements but found no difference in $\mathrm{C}+\mathrm{N}+\mathrm{O}$ between the populations. Yong et al. (2009) did, however, find a significant variation (a factor of four) in $\mathrm{C}+\mathrm{N}+\mathrm{O}$ in four RGB stars. Thus there is still uncertainty as to whether $\mathrm{C}+\mathrm{N}+\mathrm{O}$ is constant between populations or not. This is an important diagnostic since it is a very useful discriminant between possible polluters of the primordial material from which $\mathrm{N}$-rich populations form. Cluster age determinations are also very sensitive to $\mathrm{C}+\mathrm{N}+\mathrm{O}$ (Rood \& Crocker 1985; Cassisi et al. 2008). More information/observations of $\mathrm{CNO}$ are needed to clarify the situation.

In the case of $\mathrm{CN}$ band strengths, the merger scenario leads to a natural expectation that the two bimodal $\mathrm{CN}$ populations in the original clusters would also superimpose, giving a quadrimodal distribution. Thus our discovery of a quadrimodal distribution of $\mathrm{CN}$ band strengths in the RGB and AGB populations of
NGC 1851 adds further weight to the merger formation scenario for this cluster. The $\mathrm{CN}$ quadrimodality also suggests that there may be four populations with different $\mathrm{N}$ abundances in this cluster. Although $\mathrm{CN}$ is generally accepted as a proxy for $\mathrm{N}$, we note that the band strengths may also be affected by the abundances of $\mathrm{C}$ and $\mathrm{O}$. Thus, again, a complete set of (absolute) abundance observations including $\mathrm{C}, \mathrm{N}$, and $\mathrm{O}$ are needed.

With a view to gaining more information about the four $\delta \mathrm{CN}$ populations, we performed a cross-match of our sample with the Carretta et al. (2011) catalog of RGB star abundances. The cata$\log$ contains 124 stars with a range of abundance measurements for $p$-capture, $\alpha$-capture, Fe-peak, and $n$-capture elements. Our cross-match found an overlap of 10 RGB stars and one AGB star (Table 1). Not all of the Carretta et al. (2011) objects have the full set of abundance results, but for some abundances we have 7-10 stars to compare with. In Figure 4 we show various abundance ratios from Carretta et al. (2011) against our RGB $\delta \mathrm{CN}$ values. In the second panel of Figure 4, it can be seen that there appears to be no correlation between our four $\delta \mathrm{CN}$ groups and $[\mathrm{Fe} / \mathrm{H}]$. This is contrary to what we might expect from the SGB results of Gratton et al. (2012c) where it was found that the two SGB populations differ in average $[\mathrm{Fe} / \mathrm{H}]$. We note, however, that the cross-matched sample is very small, especially within each of the four subpopulations, which contain only one to four stars each. Sodium, on the other hand, shows a definite correlation with $\delta \mathrm{CN}$. This is typical of GC abundance anomalies, where $\mathrm{N}$ is higher in the stars with high Na. Barium also appears to correlate with $\delta \mathrm{CN}$. In contrast, oxygen shows little variation with $\delta \mathrm{CN}$. By using the ratio $[\mathrm{O} / \mathrm{Na}]$ the "noise" of the Fe scatter can be removed, and the relationship between $\mathrm{O}$ and $\mathrm{Na}$ is amplified. We show $[\mathrm{O} / \mathrm{Na}]$ in the top panel of Figure 4. Here, there is a striking anticorrelation, such that the $\mathrm{CN}$-weak populations show much higher $\mathrm{O} / \mathrm{Na}$ ratios than the $\mathrm{CN}$-strong populations. The lack of correlation between $\mathrm{Fe}$ (and $\mathrm{Ca}, \mathrm{Si}, \mathrm{Ti}-$ not shown) 


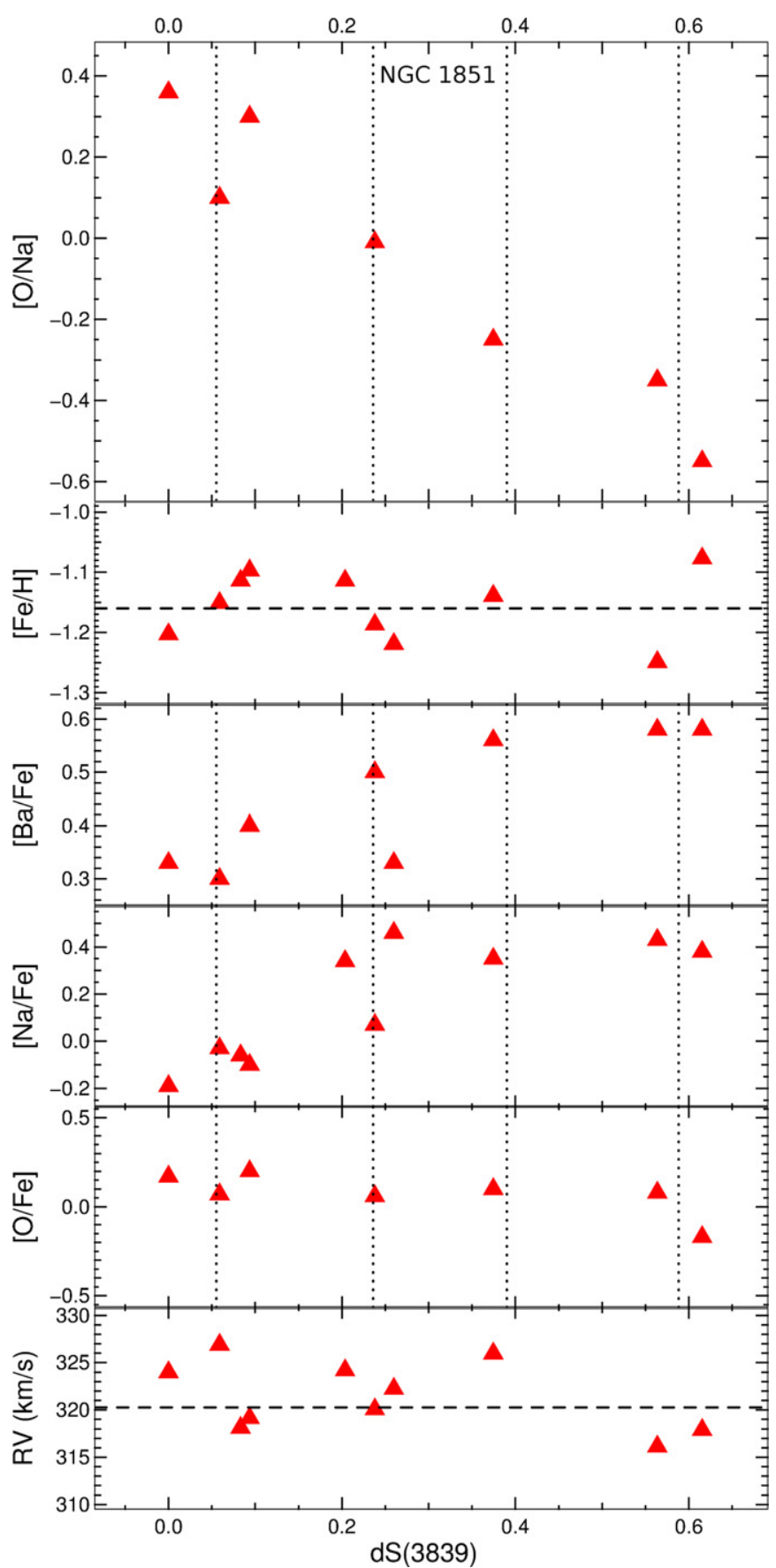

Figure 4. NGC 1851 RGB stars in common with the Carretta et al. (2011) catalog. Various abundance ratios plus radial velocity from the catalog are shown vs. our $\delta S(3839)$ values. The vertical dotted lines denote the peaks of the four $\delta \mathrm{CN}$ populations (see Figure 3 ). The horizontal lines in $[\mathrm{Fe} / \mathrm{H}]$ and radial velocity (RV) show the cluster means of -1.16 and $+320.26 \mathrm{~km} \mathrm{~s}^{-1}$, respectively (Carretta et al. 2011).

(A color version of this figure is available in the online journal.)

and the light elements $+s$-process elements suggests that the Fe-group ( $+\alpha$ capture) nucleosynthetic source(s) are separate to the light element $+s$-process source(s). AGB stars are the most likely primordial source for the $\mathrm{O}, \mathrm{Na}, \mathrm{CN}$, and $s$-process enhancements. If the four populations are indeed real, then this suggests that each population was polluted by AGB star ejecta to differing degrees. We note that the star with intermediate $[\mathrm{Ba} / \mathrm{Fe}] \sim 0.5$ is the only star in the sample with no $K$ magnitude from the Carretta et al. (2011) database and thus may be an unreliable data point. If so, then Ba would present a bimodal distribution (panel 3 of Figure 4). Importantly, each mode of Ba abundance would be associated with two $\delta \mathrm{CN}$ peaks: the two $\mathrm{CN}$-weaker populations would be Ba-poor compared to the two $\delta \mathrm{CN}$-richer populations. This could be a useful diagnostic for disentangling the multiple populations in the merger scenario. Clear bimodalities in $s$-process abundances have been reported for the RGB (Yong \& Grundahl 2008; Villanova et al. 2010) and the SGBs (Gratton et al. 2012c). Interestingly, Carretta et al. (2011) do not report a bimodality in Ba but do show that $s$-process elements are (anti)correlated with $p$-capture elements. In the bottom panel of Figure 4, we show the radial velocities for the cross-matched sample. It can be seen that only the most extreme $\delta \mathrm{CN}$ population stands out, having an average radial velocity $\sim 5 \mathrm{~km} \mathrm{~s}^{-1}$ lower than the other three populations, which might suggest this group is kinematically distinct. Again, we stress that this is a very small data set so the discussion above is only speculative. We note that our group is in the process of collecting medium resolution, broad wavelength coverage spectra using $2 \mathrm{dF} / \mathrm{AAomega}$ to complement the excellent Carretta et al. (2011) RGB data set with $\mathrm{C}$ and $\mathrm{N}$ abundances. When complete, the combined data set will allow a "holistic" analysis (including absolute abundances of $\mathrm{C}, \mathrm{N}, \mathrm{O}$, and therefore the sum $\mathrm{C}+\mathrm{N}+\mathrm{O}$ ) of the abundance and population trends for NGC 1851 red giants.

\section{SUMMARY AND CONCLUSIONS}

We have recorded a homogeneous set of spectra for 17 RGB and 21 AGB stars in the globular cluster NGC 1851. We find that the $\mathrm{CN}$ band strengths divide into four groups in both the RGB and AGB populations. This lends support to the theory that NGC 1851 formed from a merger of two clusters, since one of the expected signatures of this would be two superimposed bimodal distributions in $\mathrm{CN}$.

We cross-matched our sample with that of the high-resolution study of Carretta et al. (2011) and found a small number of stars in common. This gave us the opportunity to compare elements that typically (anti)correlate with $\mathrm{N}$ in GCs, such as $\mathrm{O}, \mathrm{Na}$, and $\mathrm{Ba}$. We found that $\mathrm{Na}$ did indeed correlate with $\delta \mathrm{CN}$. An anticorrelation $[\mathrm{O} / \mathrm{Fe}]$ was less clear but, when considering $[\mathrm{O} / \mathrm{Na}]$, it was found that there was a very strong anticorrelation. A possible correlation with $\mathrm{Ba}$ was observed. The (anti)correlations between these elements and $\delta \mathrm{CN}$ (and thus presumably $\mathrm{N}$ and hence $\mathrm{C}$ ) suggest that the material from which each of the four populations formed was polluted by AGB stars. We also speculated that the Ba distribution may be bimodal, as found in previous studies. If so, then the two $\mathrm{CN}$-weaker and two $\mathrm{CN}$-richer populations would be paired, and this may reflect a distinction between the two GCs in the merger hypothesis. It must be noted that the comparison sample is small, so strong conclusions could not be made. Large-sample, high-resolution observations combining absolute abundances of $\mathrm{C}, \mathrm{N}, \mathrm{O}, \mathrm{Fe}$, neutron-capture elements, and radial velocities in the giant branches of NGC 1851 are needed to check that there are indeed four chemically (or even kinematically) distinct populations and to determine which subpopulations are related to each other. Our group is in the process of collecting observational data to this end.

Finally we note that the AGB samples we presented here are the largest AGB samples in any GCs to date. Our finding that NGC 1851 and NGC 288 both have CN-weak-dominated AGB populations adds to a growing picture in the literature that the AGB CN distributions in GCs are different to the 
RGB distributions (Campbell et al. 2010, 2011; Lai et al. 2011; Smolinski et al. 2011; Simpson et al. 2012). For brief reviews on this topic, see Sneden et al. (2000) and Campbell et al. (2006). The current study forms part of a larger study with relatively large data sets of AGB stars to confirm this for a range of GCs (S. W. Campbell et al., in preparation).

S.W.C. acknowledges support from the Australian Research Council's Discovery Projects funding scheme (project DP1095368).

Facility: AAT

\section{REFERENCES}

Bekki, K., \& Yong, D. 2012, MNRAS, 419, 2063

Campbell, S. W., Lattanzio, J. C., \& Elliott, L. M. 2006, Mem. Soc. Astron. Ital., 77, 864

Campbell, S. W., Yong, D., Wylie-de Boer, E. C., et al. 2010, Mem. Soc. Astron. Ital., 81, 1004

Campbell, S. W., Yong, D., Wylie-de Boer, E. C., et al. 2011, in ASP Conf. Ser. 445, Why Galaxies Care about AGB Stars II: Shining Examples and Common Inhabitants, ed. F. Kerschbaum, T. Lebzelter, \& R. F. Wing (San Francisco, CA: ASP), 63

Carretta, E., Gratton, R. G., Lucatello, S., et al. 2010, ApJ, 722, L1

Carretta, E., Lucatello, S., Gratton, R. G., Bragaglia, A., \& D’Orazi, V. 2011, A\&A, 533, A69

Cassisi, S., Salaris, M., Pietrinferni, A., et al. 2008, ApJ, 672, L115

Catelan, M. 1997, ApJ, 478, L99

Cottrell, P. L., \& Da Costa, G. S. 1981, ApJ, 245, L79

Gratton, R., Sneden, C., \& Carretta, E. 2004, ARA\&A, 42, 385

Gratton, R. G., Bonifacio, P., Bragaglia, A., et al. 2001, A\&A, 369, 87

Gratton, R. G., Carretta, E., \& Bragaglia, A. 2012a, A\&AR, 20, 50

Gratton, R. G., Lucatello, S., Carretta, E., et al. 2012b, A\&A, 539, A19
Gratton, R. G., Sneden, C., Carretta, E., \& Bragaglia, A. 2000, A\&A, 354, 169 Gratton, R. G., Villanova, S., Lucatello, S., et al. 2012c, A\&A, 544, A12

Grundahl, F., Catelan, M., Landsman, W. B., Stetson, P. B., \& Andersen, M. I. 1999, ApJ, 524, 242

Han, S.-I., Lee, Y.-W., Joo, S.-J., et al. 2009, ApJ, 707, L190

Hesser, J. E. 1978, ApJ, 223, L117

Ivans, I. I., Sneden, C., Kraft, R. P., et al. 1999, AJ, 118, 1273

Kraft, R. P. 1994, PASP, 106, 553

Lai, D. K., Smith, G. H., Bolte, M., et al. 2011, AJ, 141, 62

Lardo, C., Milone, A. P., Marino, A. F., et al. 2012, A\&A, 541, A141

Lewis, I. J., Cannon, R. D., Taylor, K., et al. 2002, MNRAS, 333, 279

Martell, S. L. 2011, Astron. Nachr., 332, 467

Martell, S. L., \& Grebel, E. K. 2010, A\&A, 519, A14

Martell, S. L., Smith, G. H., \& Briley, M. M. 2008, PASP, 120, 7

Milone, A. P., Bedin, L. R., Piotto, G., et al. 2008, ApJ, 673, 241

Milone, A. P., Stetson, P. B., Piotto, G., et al. 2009, A\&A, 503, 755

Norris, J., Cottrell, P. L., Freeman, K. C., \& Da Costa, G. S. 1981, ApJ, 244, 205

Norris, J., \& Freeman, K. C. 1979, ApJ, 230, L179

Pancino, E., Rejkuba, M., Zoccali, M., \& Carrera, R. 2010, A\&A, 524, A44

Piotto, G. 2009, in The Ages of Stars IAU Symp. 258, ed. E. E. Mamajek, D. R. Soderblom, \& R. F. G. Wyse (Cambridge: Cambridge Univ. Press), 233

Rood, R. T., \& Crocker, D. A. 1985, in European Southern Observatory Conference and Workshop Proceedings, Vol. 21, ed. I. J. Danziger, F. Matteucci, \& K. Kjar (Garching, Germany: European Southern Observatory), 61

Saunders, W., et al. 2004, Proc. SPIE Conf. Ser., 5492, 389

Sharp, R., et al. 2006, Proc. SPIE Conf. Ser., 6269, 14

Simpson, J. D., Cottrell, P. L., \& Worley, C. C. 2012, arXiv:1209.0495

Skrutskie, M. F., Cutri, R. M., Stiening, R., et al. 2006, AJ, 131, 1163

Smolinski, J. P., Martell, S. L., Beers, T. C., \& Lee, Y. S. 2011, AJ, 142, 126

Sneden, C., Ivans, I. I., \& Kraft, R. P. 2000, Mem. Soc. Astron. Ital., 71,657

van den Bergh, S. 1996, ApJ, 471, L31

Villanova, S., Geisler, D., \& Piotto, G. 2010, ApJ, 722, L18

Walker, A. R. 1992, PASP, 104, 1063

Yong, D., \& Grundahl, F. 2008, ApJ, 672, L29

Yong, D., Grundahl, F., D’Antona, F., et al. 2009, ApJ, 695, L62 